\title{
Optimization of PSVR parameters of condenser based on MATLAB/Simulink simulation
}

\author{
Zhiyuan $\mathrm{Li}^{1, *}$, Kewen Wang ${ }^{1}$, and Yanhong Liu ${ }^{1}$ \\ ${ }^{1}$ School of Electrical Engineering, Zhengzhou University, Zhengzhou, China
}

\begin{abstract}
Installing a power system voltage regulator (PSVR) on the condenser can enhance the dynamic voltage control capability of the local high-voltage bus voltage, but there are still few studies on the optimization of PSVR parameters. The dynamic model of the condenser is built to determine the analytical expression between the system voltage disturbance and the change of the control target, and the influence trend of PSVR on the dynamic voltage characteristics of the condenser is analyzed. Furthermore, in the MATLAB/Simulink environment, a PSVR-containing condenser model is built, and a quantitative index for evaluating the phase-frequency characteristics of the condenser is proposed. Based on this index, the PSVR time constant is optimized through the simulation of the dynamic voltage characteristics of the condenser, and using eigenvalue root locus method combined with dynamic simulation to coordinate PSVR gain coefficient, achieve PSVR parameter optimization. The simulation results under the single-machine and three-machine systems show that after the PSVR parameter optimization, the voltage oscillation mode of the condenser is effectively improved.
\end{abstract}

\section{Introduction}

Synchronous condensers can provide reactive power support in the event of system failures, inhibit DC receiving end commutation failure, and have good reactive power output and transient voltage response characteristics, so they have been widely used in UHV DC transmission systems[1-4]. Installing a Power System Voltage Regulator (PSVR) can maintain the high-voltage bus voltage at a higher level. PSVR introduces the generator high-voltage side voltage to participate in the automatic voltage excitation regulator (Automatic Voltage Regulator, AVR) control, it can keep the bus voltage on the high-voltage side of the main transformer of the condenser at a higher level, which can further improve the dynamic voltage characteristics of the condenser.

In order to better improve the system voltage characteristics, many scholars have launched a series of studies on PSVR[5-7]. But most of the above documents are based on conventional generators for PSVR parameter design, without considering the possible impact of adjusting the condenser on the system, so this article considers using PSVR for adjusting the condenser, and combining the dynamic characteristics of the adjusting condenser to optimize the design of PSVR parameters.

In summary, this article proposes a method to optimize PSVR parameters based on the dynamic voltage characteristics of the condenser. In the MATLAB/Simulink environment, a single-machine infinity and a three-machine system are built, an index to measure the phase shift of the dynamic voltage characteristics of the condenser is proposed, the lead/lag time constant is optimized, and the eigenvalue root locus method is used to coordinate the gain coefficient. The optimization method is verified by simulation.

\section{Condenser and PSVR}

The condenser can provide sufficient dynamic reactive power support while the system is running in a steady state. The control signal of the power system voltage regulator (PSVR) is introduced into the adjustable condenser excitation system to further improve the dynamic voltage characteristics of the adjustable condenser[8].

PSVR takes the deviation of the high-voltage bus voltage on the main transformer side as the input signal, and after amplification, lead/lag, and output limiting, the result is output to the excitation system to maintain the high-voltage bus voltage at a higher level. The PSVR control block diagram is shown in figure 1. In the figure: $\Delta V$ is the deviation between the high-voltage bus voltage reference value and the measured value, $K_{\mathrm{v}}$ is the gain coefficient, $T_{\mathrm{v}}$ is the transmitter time constant, $T_{\mathrm{q} 1}, T_{\mathrm{q} 1}, T_{\mathrm{q} 2}$, $T_{\mathrm{q} 2}, T_{\mathrm{q} 3}, T_{\mathrm{q} 3}$ are the time constants of the lead/lag link, $\Delta$ $V_{\mathrm{S}}, \Delta V_{\mathrm{Smax}}$ and $\Delta V_{\mathrm{Smin}}$ are the PSVR output amplitude and its limit value respectively.

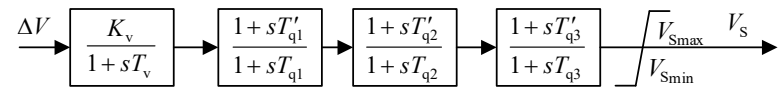

Fig. 1. PSVR control block diagram.

\footnotetext{
*Corresponding author: rewter456@163.com
} 
Take the actual power system as an example, using the excitation voltage control shown in figure 2 . The tuned condenser with a rated capacity of $300 \mathrm{Mvar}$ and a rated voltage of $20 \mathrm{kV}$ is connected to the $525 \mathrm{kV}$ bus through a step-up transformer. Since there is a big difference between the operating mode of the condenser and the generator, it is necessary to reasonably optimize and adjust the PSVR parameters according to the operating conditions of the condenser.

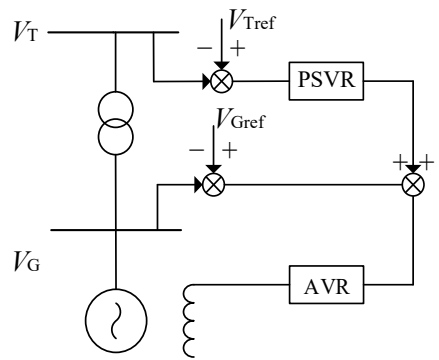

Fig. 2. Excitation voltage control

At present, the PSVR parameters are usually tuned by a parameter method similar to the power system stabilizer (PSS), that is, the phase time constant is tuned by the phase compensation method, and the gain coefficient is determined by the root locus method. The operating mode of the condenser is different from the ordinary generator, and only outputs reactive power. It is necessary to analyze the dynamic reactive voltage characteristics of the condenser, and optimize and adjust the PSVR parameters accordingly.

\section{Analysis of condenser dynamic voltage characteristics}

At present, there are few theoretical analysis on the dynamic reactive voltage characteristics of the condenser before and after the installation of PSVR. Analyze the influence of the infinite bus voltage change on the highvoltage bus voltage on the basis of the single-machine infinite bus model. The analysis of PSVR control on voltage stability is as follows.Assuming that the condenser is a sixth-order model[9], the linearized relationship is as follows:

$$
\begin{gathered}
\Delta E_{\mathrm{d}}^{\prime \prime}=\left[\Delta E_{\mathrm{d}}^{\prime}+\left(X_{\mathrm{q}}^{\prime}-X_{\mathrm{q}}^{\prime \prime}\right) \Delta I_{\mathrm{q}}\right] /\left(1+T_{\mathrm{q} 0}^{\prime \prime} s\right) \\
\Delta E_{\mathrm{q}}^{\prime \prime}=\left[\Delta E_{\mathrm{q}}^{\prime}-\left(X_{\mathrm{d}}^{\prime}-X_{\mathrm{d}}^{\prime \prime}\right) \Delta I_{\mathrm{d}}\right] /\left(1+T_{\mathrm{d} 0}^{\prime \prime} s\right) \\
\Delta E_{\mathrm{d}}^{\prime}=\left[\left(X_{\mathrm{q}}-X_{\mathrm{q}}^{\prime}\right) \Delta I_{\mathrm{q}}\right] /\left(1+T_{\mathrm{q} 0}^{\prime} s\right) \\
\Delta E_{\mathrm{q}}^{\prime}=\left[\Delta E_{\mathrm{fd}}-\left(X_{\mathrm{d}}-X_{\mathrm{d}}^{\prime}\right) \Delta I_{\mathrm{d}}\right] /\left(1+T_{\mathrm{d} 0}^{\prime} s\right) \\
\Delta \delta=\omega_{0}\left(\Delta \omega-\Delta \omega_{\mathrm{ref}}\right) / s \\
\Delta \omega=\left(\Delta P_{\mathrm{m}}-\Delta P_{\mathrm{e}}\right) /\left(T_{\mathrm{J}} s\right) \\
\Delta V_{\mathrm{g}}=V_{\mathrm{d}} \Delta V_{\mathrm{d}} / V_{\mathrm{g}}+V_{\mathrm{q}} \Delta V_{\mathrm{q}} / V_{\mathrm{g}} \\
\Delta V_{\mathrm{d}}=\Delta E_{\mathrm{d}}^{\prime \prime}-R_{\mathrm{a}} \Delta I_{\mathrm{d}}+X_{\mathrm{q}}^{\prime \prime} \Delta I_{\mathrm{q}} \\
\Delta V_{\mathrm{q}}=\Delta E_{\mathrm{q}}^{\prime \prime}-X_{\mathrm{d}}^{\prime \prime} \Delta I_{\mathrm{d}}-R_{\mathrm{a}} \Delta I_{\mathrm{q}} \\
\Delta P_{\mathrm{e}}=\left(V_{\mathrm{d}}+2 R_{\mathrm{a}} I_{\mathrm{d}}\right) \Delta I_{\mathrm{d}}+\left(V_{\mathrm{q}}+2 R_{\mathrm{a}} I_{\mathrm{q}}\right) \Delta I_{\mathrm{q}} \\
+I_{\mathrm{q}} \Delta V_{\mathrm{q}}+I_{\mathrm{d}} \Delta V_{\mathrm{d}}
\end{gathered}
$$

According to the derivation of the relationship of the sixth-order model of the condenser, we can get:

$$
\begin{gathered}
\left\{\begin{array}{l}
\Delta V_{\mathrm{d}}=G_{1} \Delta I_{\mathrm{d}}+G_{2} \Delta I_{\mathrm{q}} \\
\Delta V_{\mathrm{q}}=G_{3} \Delta I_{\mathrm{d}}+G_{4} \Delta I_{\mathrm{q}}
\end{array}\right. \\
\Delta \delta=G_{5} \Delta I_{\mathrm{x}}+G_{6} \Delta I_{\mathrm{y}}+G_{7} \Delta V_{\mathrm{x}}+G_{8} \Delta V_{\mathrm{y}}
\end{gathered}
$$

Transformed by Parker, we can get:

$$
\begin{gathered}
\left\{\begin{array}{c}
\Delta I_{\mathrm{d}}=\Delta I_{\mathrm{x}} \sin \delta-\Delta I_{\mathrm{y}} \cos \delta+I_{\mathrm{q}} \Delta \delta \\
\Delta I_{\mathrm{q}}=\Delta I_{\mathrm{x}} \cos \delta+\Delta I_{\mathrm{y}} \sin \delta-I_{\mathrm{d}} \Delta \delta
\end{array}\right. \\
\left\{\begin{array}{l}
\Delta V_{\mathrm{x}}=\Delta V_{\mathrm{q}} \cos \delta+\Delta V_{\mathrm{d}} \sin \delta-V_{\mathrm{y}} \Delta \delta \\
\Delta V_{\mathrm{y}}=\Delta V_{\mathrm{q}} \sin \delta-\Delta V_{\mathrm{d}} \cos \delta+V_{\mathrm{x}} \Delta \delta
\end{array}\right.
\end{gathered}
$$

Equations (11), (12), (13) and (14) are combined to get

$$
\left\{\begin{array}{l}
\Delta V_{\mathrm{x}}=G_{9} \Delta I_{\mathrm{x}}+G_{10} \Delta I_{\mathrm{y}} \\
\Delta V_{\mathrm{y}}=G_{11} \Delta I_{\mathrm{x}}+G_{12} \Delta I_{\mathrm{y}}
\end{array}\right.
$$

In a single-machine infinite bus system, the relationship between the high-voltage bus voltage and the terminal voltage and current is:

$$
\begin{aligned}
\Delta V_{\mathrm{T}}= & \frac{V_{\mathrm{Tx}}}{V_{\mathrm{T}}}\left(\Delta V_{\mathrm{x}}+R_{\mathrm{T}} \Delta I_{\mathrm{x}}-X_{\mathrm{T}} \Delta I_{\mathrm{y}}\right)+ \\
& \frac{V_{\mathrm{Ty}}}{V_{\mathrm{T}}}\left(\Delta V_{\mathrm{y}}+R_{\mathrm{T}} \Delta I_{\mathrm{y}}-X_{\mathrm{T}} \Delta I_{\mathrm{x}}\right)
\end{aligned}
$$

The relationship between the high-voltage bus voltage and the infinite bus voltage is:

$$
\Delta \dot{V}_{\mathrm{s}}=\Delta \dot{V}_{\mathrm{T}}-\mathrm{j} \Delta \dot{I} X_{\mathrm{s}}
$$

Equations (15), (16) and (17) can be combined

$$
\begin{gathered}
\Delta \dot{V}_{\mathrm{T}}=G \Delta \dot{I} \\
\Delta \dot{V}_{\mathrm{T}}=\frac{G}{G-X_{\mathrm{s}}} \Delta \dot{V}_{\mathrm{s}}
\end{gathered}
$$

After adding PSVR, for the convenience of expression, add 0 to the subscripts of the terminal voltage deviations $\Delta V_{\mathrm{x}}$ and $\Delta V_{\mathrm{y}}$ obtained when PSVR is not added, which can be obtained after simplification according to the sixthorder generator model and the open-loop relationship:

$$
\left\{\begin{array}{l}
\Delta V_{\mathrm{x}}=\Delta V_{\mathrm{x} 0}+H_{1} \Delta V_{\mathrm{T}} \\
\Delta V_{\mathrm{y}}=\Delta V_{\mathrm{y} 0}+H_{2} \Delta V_{\mathrm{T}}
\end{array}\right.
$$

In the same way, formula (16), formula (17) and formula (20) are combined to get:

$$
\begin{gathered}
\Delta \dot{V}_{\mathrm{T}}=H \Delta \dot{I} \\
\Delta \dot{V}_{\mathrm{T}}=\frac{H}{H-X_{\mathrm{s}}} \Delta \dot{V}_{\mathrm{s}}
\end{gathered}
$$

In Equation (1)-Equation (22), $G_{1}-G_{12}, G, H_{1}, H_{2}$ and $H$ are the expressions of generator parameters and transformer parameters, $X_{\mathrm{S}}$ is the line impedance from the high-voltage side of the transformer to the infinite bus, and the other variables are the same as conventional usage Unanimous. According to equations (19) and (22), the changes in the system voltage characteristics before and after PSVR can be obtained. 


\section{PSVR parameter optimization}

Installing PSVR to the condenser can improve the dynamic voltage characteristics of the condenser. According to equations (19) and (22), the corresponding dynamic model can be established to optimize the parameters of the PSVR and improve system stability. The optimization and adjustment of PSVR parameters will be carried out in three stages:

- According to the expression of the dynamic voltage characteristics of the condenser, build a condenser dynamic model based on MATLAB/Simulink tools, obtain the amplitude-frequency phase-frequency characteristic curve between the high-voltage bus voltage and the infinite bus voltage before and after the PSVR is installed, and propose a measure of the phasefrequency characteristic The index of the degree of phase shift, with the goal of minimizing the proposed index, adjust the lead/lag time constant.The smaller the phase shift, the smaller the impact on the power angle stability. For the convenience of description, the absolute value of the phase-frequency characteristic curve is taken, and the sum of the logarithmic form is taken as an index to measure the degree of the phasefrequency characteristic phase shift. This index can reflect the degree of coupling of existing power and reactive power. The smaller the value, the smaller the impact on active power.

$$
\theta=\sum_{i=1}^{N} \ln \left(\left|\theta_{\mathrm{i}}\right|\right)
$$

In the formula, $N$ is 30 , and $\theta_{1}$ is the value of the $\mathrm{i}$-th power angle.

- Adjust the PSVR gain coefficient through the characteristic root locus method to determine the adjustment range of the gain coefficient. Increase the gain coefficient from 0 , calculate the eigenvalue of the system, and judge the stability of the system through the real part of the eigenvalue. Observe the reactive power output of the condenser and the waveform of the highvoltage bus voltage. By processing the waveform, the real and imaginary parts of the eigenvalues and the damping ratio can be obtained to determine whether the gain coefficient is appropriate.

- Taking into account the influence of the multi-machine system, further adjust the PSVR parameters in the threemachine system simulation model. Substitute the PSVR parameters selected in the single-machine system into the three-machine system, check whether the parameters are reasonable, and adjust the parameters again.

The multi-machine system used in the actual project may be more complicated. In the future work, a more complicated system will be built to verify whether the proposed method is reasonable.

\section{Simulation analysis}

This paper builds a single-machine infinite bus system model and a three-machine system model based on MATLAB/Simulink simulation software.

\subsection{Generator dynamic voltage characteristics}

The condenser dynamic model is used to analyze the influence of PSVR on the dynamic voltage characteristics of the condenser before and after installation.

When PSVR is not installed, the $V_{\mathrm{T}}-V_{\mathrm{S}}$ amplitudefrequency phase-frequency curve is shown in figure 3 , and the $\theta$ value is 39.94 .

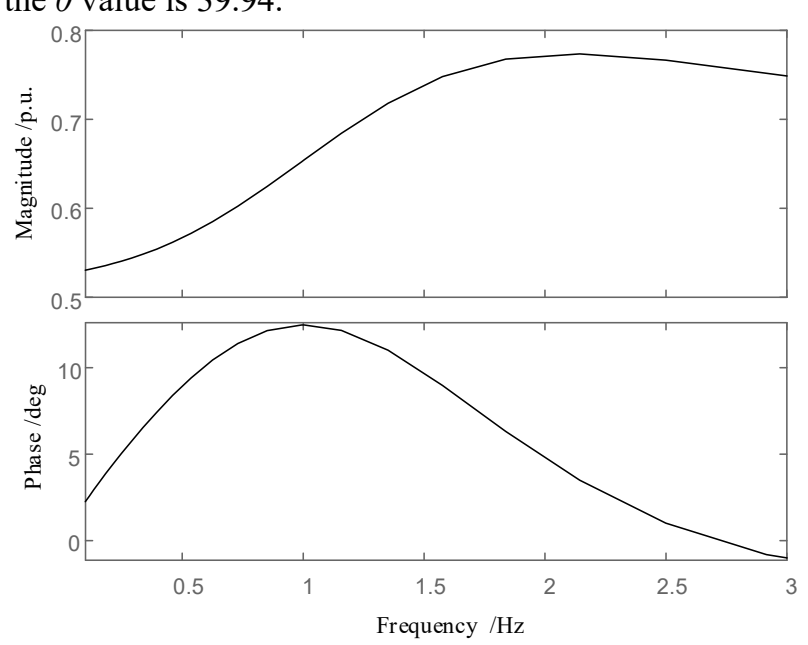

Fig. 3. $V_{\mathrm{T}}-V_{\mathrm{S}}$ amplitude-frequency phase-frequency characteristic curve example

When PSVR is installed, the actual values of PSVR parameters are: $K_{\mathrm{v}}=1.2, T_{\mathrm{v}}=0.1, T_{\mathrm{q} 1}=0.5, T_{\mathrm{q} 1}=0.2$, and the other parameters are all 0 . The reactive power output is 1p.u., and its $V_{\mathrm{T}}-V_{\mathrm{S}}$ characteristic is shown in figure 4 . At this time, the value of $\theta$ increases to 83.35.

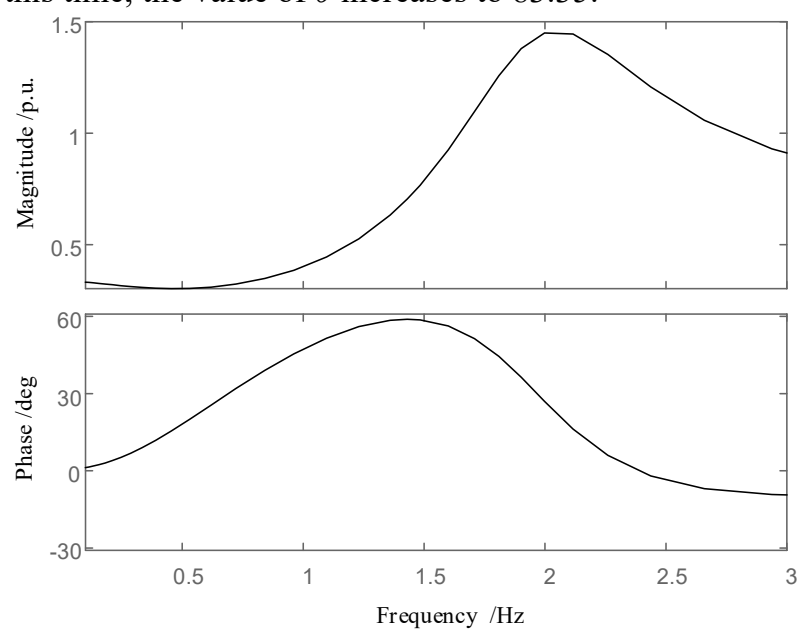

Fig. 4. $V_{\mathrm{T}}-V_{\mathrm{S}}$ amplitude-frequency phase-frequency characteristic curve when PSVR is assembled

It can be seen from figure 4 that When PSVR is installed,, the voltage power angle changes greatly. The installation of PSVR will affect the voltage characteristics of the system, so it is necessary to adjust the PSVR parameters reasonably to reduce its impact on the voltage power angle.

\subsection{PSVR lead/lag time constant adjustment}

Through the simulation model of the single-machine infinite bus system, On the basis of the PSVR parameters in the previous section, change the time constants of the 
two lead/lag links after PSVR to reduce its influence on the power angle characteristics. After many simulation analyses and comparisons, when $T_{\mathrm{q} 2}=0.1, \quad T^{\prime}{ }_{\mathrm{q} 2}=1.4$, $T_{\mathrm{q} 3}=0.1, \quad T_{\mathrm{q} 3}=1.5$, the amplitude-frequency phasefrequency characteristic curve is shown in figure 5 . The $\theta$ value dropped from 83.35 to 45.41 .
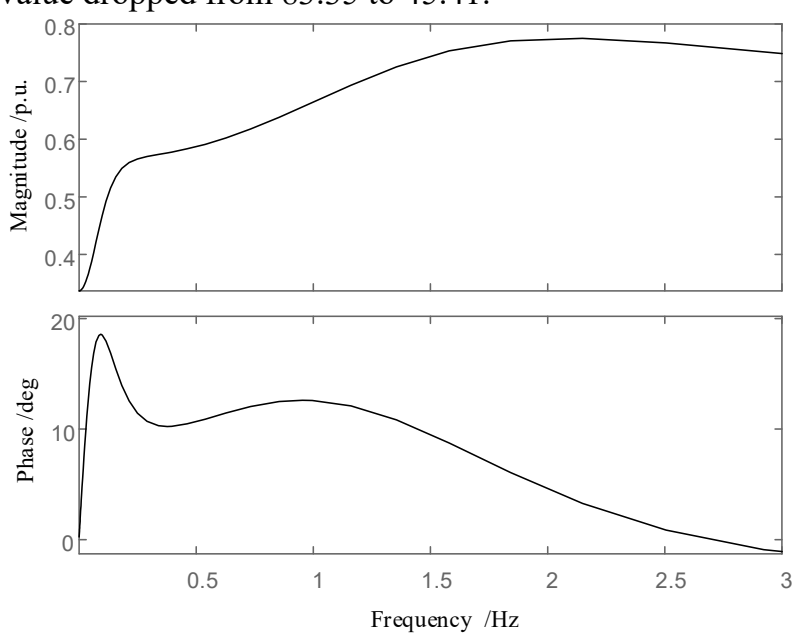

Fig. 5. $V_{\mathrm{T}}-V_{\mathrm{S}}$ amplitude-frequency phase-frequency characteristic curve

\subsection{PSVR lead/lag time constant adjustment}

After adjusting the lead/lag time constant, keep the value of the time constant unchanged and adjust the PSVR gain coefficient. In a single-machine infinite bus system, the PSVR gain coefficient is increased from 0 , and the system eigenvalue is calculated until the real part of the eigenvalue appears 0 . The gain factor at this time is the critical gain of PSVR, and 1/8-1/3 of its value is taken as the best gain factor.

When the gain coefficients are $10,45.5$, and 46.5 , the high-voltage bus voltage waveform is shown in figure 6 , and the corresponding eigenvalue damping ratio is shown in table 1.

Table 1. Comparison of eigenvalues under different gain coefficients

\begin{tabular}{|c|c|c|c|}
\hline Gain factor & $\begin{array}{c}\text { Eigenvalue } \\
\text { real part }\end{array}$ & $\begin{array}{c}\text { Imaginary } \\
\text { part }\end{array}$ & $\begin{array}{c}\text { Damping } \\
\text { ratio }\end{array}$ \\
\hline$K_{\mathrm{V}}=10$ & -0.4624 & 8.78 & 0.0526 \\
\hline$K_{\mathrm{V}}=45.5$ & -0.0206 & 9.60 & 0.0021 \\
\hline$K_{\mathrm{V}}=46$ & 0.0039 & 9.599 & -0.00041 \\
\hline$K_{\mathrm{V}}=46.5$ & 0.0345 & 9.307 & -0.00371 \\
\hline
\end{tabular}

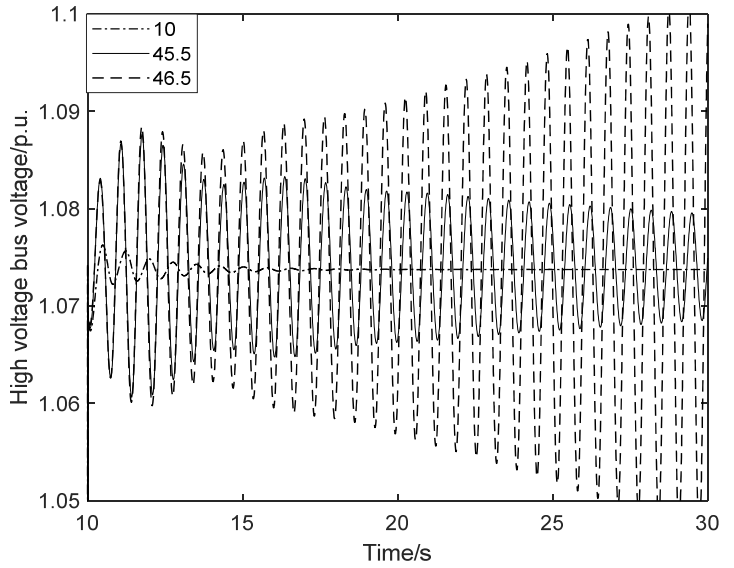

Fig. 6. High voltage bus voltage waveform under different gain coefficients

It can be obtained from figure 6 and table 1 that the PSVR gain coefficient should not be greater than 46 . When the gain coefficient is 46 , the real part of the eigenvalue appears non-negative, and the system is unstable. Therefore, the value range of the gain coefficient should be 5.75-15.3, and the optimal gain coefficient is set to 10 .

\subsection{Three-machine system inspection parameters}

Substitute the optimized PSVR parameters under the single-machine infinite bus system into the three-machine system, the high-voltage bus voltage and reactive power waveforms of the simulated condenser are shown in figure 7. When the gain coefficient is 46.5 , the real part of the eigenvalue of the three-machine system is still negative. When the PSVR gain coefficient is increased to 48.5 , the real part of the eigenvalue appears positive. The critical gain of a three-machine system and a single-machine infinite bus system is slightly different, but it is within an acceptable range. In the three-machine system, when the generator is disturbed, the generator can provide part of the voltage regulation capability, so the PSVR critical gain coefficient will be larger than that in the single-machine system.

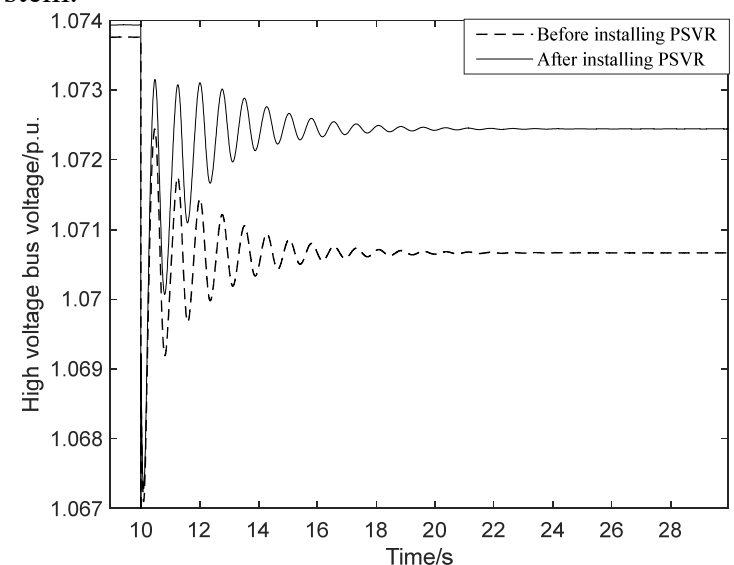

Fig. 7. Three-machine system reactive power and high-voltage bus voltage waveform when $K \mathrm{v}=10$ 


\section{Conclusion}

With reference to the actual system data, taking the singlemachine infinite bus system as the research object, the dynamic voltage characteristics of the condenser are deduced, and a PSVR parameter optimization method that is conducive to voltage stability is proposed. The PSVR lead/lag time constant is adjusted by calculating the phasefrequency characteristic phase shift index, and the PSVR gain coefficient is adjusted according to the eigenvalue root locus method. The simulation models of the singlemachine infinite bus system and the three-machine system are built in MATLAB/Simulink, and the optimization method is verified through simulation. The results show that the optimized parameters can effectively improve the voltage oscillation mode of the condenser.

\section{Acknowledgments}

The National Natural Science Foundation of China (61473265)

\section{References}

1. L. Richard, N. A. Masood, T. K. Saha, W. Tushar, H. Gu, IEEE Access, 8(2020)

2. J. Jia, G. Yang, A. H. Nielsen, V. Gevorian, IEEE Trans. Power Delivery, 34, 5(2019)

3. J. Jia, G. Yang, A. H. Nielsen, P. R. Hansen, IEEE Trans. Ind. Electron, 66, 2(2019)

4. M. M. Alam, H. Leite, N. Silva, Electric Power Syst.Res, 152(2017)

5. A. Zou, J. Leng, X. He, Y. Zu, C. D. Gill, V. J. Reddi, X. Zhang, IEEE Trans. Comput. Aided Des. Integr. Circuits Syst., 39(2020)

6. X. Chen, H. Yu, J. Lu, Y. Gu, X. Wu, C. Cao, Electric Power Systems, 36, 3(2012)

7. X. Chen, G. Qin, M. Yang, L. Zhao, R. Chen, G. Xie, Electric Power, 52,3(2019)

8. J. W. Liu, S. S. Choi, S. Chen, IEEE Trans. Power Delivery, 18, 4(2003)

9. W. Huang, D. J.Hill, X. Zhang, IEEE Trans. Power Syst, 35, 4(2020) 